Aim of the study: Helical tomotherapy is one of the methods of radiotherapy. This method enables treatment implementation for a wide spectrum of clinical cases. The vast array of therapeutic uses of helical tomotherapy results directly from the method of dose delivery, which is significantly different from the classic method developed for conventional linear accelerators. The paper discusses the method of dose delivery by a tomotherapy machine. Moreover, an analysis and presentation of treatment plans was performed in order to show the therapeutic possibilities of the applied technology. Dose distributions were obtained for anaplastic medulloblastoma, multifocal metastases to brain, vulva cancer, tongue cancer, metastases to bones, and advanced skin cancer. Tomotherapy treatment plans were compared with conventional linear accelerator plans. Results: Following the comparative analysis of tomotherapy and conventional linear accelerator plans, in each case we obtained the increase in dose distribution conformity manifested in greater homogeneity of doses in the radiation target area for anaplastic medulloblastoma, multifocal metastases to brain, vulva cancer, metastases to bones, and advanced skin cancer, and the reduction of doses in organs at risk (OAR) for anaplastic medulloblastoma, vulva cancer, tongue cancer, and advanced skin cancer. The time of treatment delivery in the case of a tomotherapy machine is comparable to the implementation of the plan prepared in intensity-modulated radiotherapy (IMRT) technique for a conventional linear accelerator. In the case of tomotherapy the application of a fractional dose was carried out in each case during one working period of the machine. For a conventional linear accelerator the total value of the fractional dose in the case of anaplastic medulloblastoma and metastases to bones was delivered using several treatment plans, for which a change of set-up was necessary during a fraction.

Conclusion: The obtained results confirm that tomotherapy offers the possibility to obtain precise treatment plans together with the simplification of the therapeutic system.

Key words: helical tomotherapy, IMRT, treatment planning, evaluation of the dose distribution.

\section{Tomotherapy - a different way of dose delivery in radiotherapy}

\author{
Tomasz Piotrowski ${ }^{12}$, Małgorzata Skórska², Agata Jodda², \\ Adam Ryczkowski², Joanna Kaźmierska ${ }^{13}$, Krystyna Adamska ${ }^{4}$, \\ Aldona Karczewska-Dzionk ${ }^{5}$, Małgorzata Żmijewska-Tomczak ${ }^{6}$, \\ Hanna Włodarczyk ${ }^{7}$
}

1Department of Electroradiology, Poznan University of Medical Sciences, Poland 2Department of Medical Physics, Greater Poland Cancer Centre, Poznań, Poland 3Department of Radiotherapy II, Greater Poland Cancer Centre, Poznań, Poland 4Second Radiotherapy Ward, Greater Poland Cancer Centre, Poznań, Poland 5Third Radiotherapy Ward, Greater Poland Cancer Centre, Poznań, Poland ${ }^{6}$ Department of Radiotherapy I, Greater Poland Cancer Centre, Poznań, Poland ${ }^{7}$ Gynaecological Radiotherapy Ward, Greater Poland Cancer Centre, Poznań, Poland

\section{Introduction}

In the case of classic radiation therapy, delivered by conventional medical accelerators, the ionizing radiation beam is of divergent character. In other words, the ionizing radiation is emitted from the source to the pyramid-shaped area. In the simplest case, when the radiation beam is shaped by primary field collimators (jaws) alone, a rectangle is the base of the pyramid. The base of the pyramid, perpendicular to the beam's axis, delineates the shape of the treatment field, which usually fully covers a radiotherapy field defined by the clinician [1]. Thus, the radiation dose is delivered to the whole treatment area in a volumetric manner.

In order to conform the dose to the radiation treatment area most precisely, the ionizing radiation dose may be delivered either during the movement of the machine's gantry around the longitudinal axis of a patient (rotary technique) or through the application of several fixed positions of the gantry [2]. Both these methods offer the possibility of using additional modifiers altering the shape of applied treatment fields.

The multi-leaf collimator (MLC) is one of the basic modifiers [3]. The use of the MLC allows for both static modulation of a treatment field, which remains fixed during the emission of ionizing radiation [4, 5], and for dynamic modulation, causing changes in the shape of the field in the course of radiation emission [6]. The latter solution enables the delivery of intensity-modulated radiotherapy (IMRT) [7-9].

The IMRT technique is a basic method used in modern radiotherapy. In the typical form of IMRT, several (generally from 5 to 9) fixed settings of the gantry are used [10-12]. In recent years, the manufacturers of the radiation therapy equipment (Varian, Elekta), through implementing technical advances, have enabled the integration of classic rotary techniques with IMRT, which has resulted in great popularity of VMAT and Rapid Arc [13-17]. The method of dose delivery to the irradiated volume in the case of helical tomotherapy differs significantly from the volumetric method employed by conventional linear accelerators [18].

The term "helical tomotherapy" had already been coined by the end of the 1980s. After 15 years of intensive studies and technological advances, tomotherapy machines have been implemented at cancer centres. The prototype of the machine was completed in 2001 at Wisconsin University. A year later, the first patients underwent treatment [19]. At present, tomotherapy is not only an acknowledged and widely accepted radiotherapeutic method but 
is also considered to be one of the most advanced and precise methods of dose delivery.

In 2009 the first helical tomotherapy machine was installed in the Greater Poland Cancer Centre. The paper discusses the method of emission of ionizing radiation and the related method of dose delivery by the tomotherapy machine. Moreover, in order to present therapeutic possibilities of the applied technology, treatment plans of selected clinical cases treated with tomotherapy are presented and reviewed. Ad ditionally, tomotherapy treatment plans are compared with the plans prepared for a conventional linear accelerator.

\section{Material and methods}

\section{Tomotherapy machine - construction and operation}

A tomotherapy unit resembles a computed tomography machine. The casing fits a rotating ring into which a treatment table with a patient slides. A rotary movement of the gantry is combined with a progressive movement of the treatment table and thus the source of radiation rotates around the patient along a helical path (helical tomotherapy) [20]. It is also possible to deliver radiation by means of a direct method (direct tomotherapy) in which first the angles of dose delivery projection are determined and next the treatment is performed according to specific assumptions [21].

The source of ionizing radiation is a linear accelerator whose nominal voltage is $6 \mathrm{MV}$. The emitted photon beam has the shape of a fan, $40 \mathrm{~cm}$ wide and $1 \mathrm{~cm}, 2.5 \mathrm{~cm}$ or $5 \mathrm{~cm}$ thick (measured in the isocentre). The latest version of a tomotherapy system enables to use both treatment table motion variable in time and dynamic jaws whose width can continuously change in the range of $1 \mathrm{~cm}$ to $5 \mathrm{~cm}$ [22]. Sixty-four collimator leaves either block or let in radiation in individual parts of the fan.

The collimator works in a binary fashion. In other words, an individual leaf can be fully open or fully closed and thanks to the pneumatic drive its action is very quick: the full opening-closure cycle lasts only 20 ms [23].

The application of the matrix of detectors located on the opposite side of the ring makes it possible for a tomotherapy unit to operate like a computed tomography scanner. This allows one to verify the patient's position on a treatment table on a daily basis by comparing MVCT (megavoltage CT) scans with computed tomography images, used for radiotherapy planning. The nominal voltage of a linear accelerator in the imaging mode is lowered to $3.5 \mathrm{MV}$, which gives images of better quality. Moreover, the fact that on delivering the dose ranging from 0.5 cGy to 3 cGy (comparable with diagnostic dose) it was possible to obtain MVCT scans is of paramount importance. In the case of a highenergy fan beam the impact of dispersed radiation registered by detectors is negligible. Therefore, as with classic computed tomography, it is possible to determine a direct and more importantly a stable relation between grey scale expressed in $\mathrm{HU}$ and the density of the imaged structures. This relation makes it possible to reconstruct, on the prepared MVCT scans, the dose distribution which is delivered during a session, the dose planned earlier or the difference between them [24, 25].

\section{Tomotherapy treatment planning}

The system of treatment planning for tomotherapy fully makes use of the idea of inverse planning $[26,27]$. While preparing a treatment plan the number of treatment beams and their projection angles are not defined. However, the parameters determining the method of dose delivery such as jaws width, pitch, and the value of the modulation factor should be defined. The width of jaws and pitch are fixed parameters, which should be determined before the process of optimization starts. The value of pitch factor is determined by the ratio of the gantry rotation and the table movement expressed in the units of field width. In the case of helical tomotherapy the value of this parameter should be smaller than 1 . This means that the fields formed by consecutive rotations overlap: the smaller the pitch value, the bigger the degree of overlapping. In order to obtain the most homogeneous dose distribution, including over the areas located outside the isocentre, the pitch parameter should equal $0.86 / n$, for $n=1,2,3, \ldots$ [28]. The MF parameter is defined as the relation of the maximum leaf opening time to the average leaf opening time. MF may be altered during the optimization of a treatment plan. In the course of treatment plan preparation one should remember to define the method of normalization and the parameters of the expected dose distribution for the individual structures and their relative significance.

The next stage of planning, typical of tomotherapy, is beamlet calculations. A beamlet is an element of a beam emitted through a single open leaf during one projection. For the needs of the optimization algorithm, the continuous rotation of the gantry around the patient is divided into 51 projections (the angles of beam incidence). Each contains 64 beamlets corresponding to 64 collimator leaves, which amounts to 3,264 beams for each gantry rotation. Next, each of the beamlets is divided into, at least, as many elements as there are voxels to which radiation is transported by it. Thus, a computer determines the set of beams for each volume element. The optimization algorithm, by prolonging or shortening the leaf opening time, changes the dose delivered to individual voxels.

A patient is irradiated from all sides, which allows one to obtain a homogeneous dose distribution in a tumour, with a large gradient at its edges, and enables reduction of the dose absorbed by adjoining healthy tissues. The process of treatment plan optimization resembles those which were used in computer systems of treatment planning (Eclipse, Helax, Ocentra) which operate conventional linear accelerators. The specificity of the irradiation method with the use of a tomotherapy machine enables one to carry out a full treatment plan during one beam release. In one session both a very small area (uni- or multifocal stereotactic treatment, where the minimum treatment field size is $5 \mathrm{~mm} \times 6.25 \mathrm{~mm}$ ) and a very large area (e.g. cranio-spinal irradiation; maximum treatment field size $-400 \mathrm{~mm} \times 1600 \mathrm{~mm}$ ) can be irradiated. What is more, no change in the patient's position or combining the fields emitted from two or more beams is required.

\section{Examples of clinical deliveries}

With the aim of presenting a spectrum of helical tomotherapy possibilities, we chose six clinical cases repre- 
senting irradiation of the following: 1 - anaplastic medulloblastoma, 2 - multifocal metastases to brain, 3 - vulva cancer, 4 - tongue cancer, 5 - metastases to bones, 6 - advanced skin cancer. For cases 2, 3, 6 the original treatment plan was prepared for radiotherapy delivery performed by a conventional linear accelerator. However, due to the unsatisfactory dose distribution or problems with the realization of the plan by the machine (gantry-table collisions for planned treatment fields), an attempt was made to deliver treatment using helical tomotherapy. For cases 1, 4, 5 helical tomotherapy was the chosen method. In order to compare the obtained dose distributions with the distributions obtained for a conventional linear accelerator, three independent treatment plans were prepared (three people preparing plans who did not know results for helical tomotherapy). Next, among the prepared plans, we chose the best in terms of homogeneity of dose distributions in planning target volume (PTV), and reduction of dose in critical organs. The chosen treatment plan was compared with the plan for helical tomotherapy.

Below, we briefly discuss the clinical cases and present recommendations concerning the total dose size, its fractionation, regions of irradiation and critical organs.

\section{Case 1 (medulloblastoma)}

History: a ten-year-old girl, diagnosed with a tumour located in the cerebellar vermis with metastasis to the spinal cord. The patient underwent non-radical surgery in the area of the medulla oblongata. The tumour also infiltrated the brain stem. Stage IV of clinical progression according to WHO.

Therapeutic strategy: Three-stage treatment involved: (a) delivery of $55.11 \mathrm{~Gy}$ dose to the region of residual lesion adjoining brain stem in 36 fractions, (b) delivery of 41.75 Gy dose to the metastatic area (thoracic part of the spinal cord) in 25 fractions, and (c) delivery of 35.07 Gy dose in 21 fractions to the whole craniospinal area (spinal cord and brain). The critical organs (OAR) were the following: brain stem, eyes, lenses, optic nerves, optic chiasm, left and right internal ear, parotid glands, larynx, thyroid, lungs, heart, kidneys, liver, pancreas, stomach, spleen and intestines.

\section{Case 2 (multifocal brain metastases)}

History: 56-year-old woman diagnosed with nine metastatic lesions from primary breast cancer. The patient was irradiated for the whole brain; a dose of 40 Gy was delivered in 20 fractions. Time since completion of the therapy 18 months.

Therapeutic strategy: irradiation of the brain taking into account escalation of the dose over metastatic areas. The following fractionation scheme was chosen: 15 Gy dose to the whole brain delivered in 10 fractions with simultaneous escalation of the dose to the metastatic lesion to $30 \mathrm{~Gy}$. Critical organs: eyes, lenses, optic nerves, optic chiasm.

\section{Case 3 (vulva cancer)}

History: A 65-year-old woman diagnosed with tumour of the vagina underwent surgery in 2008 , to remove a vaginal tumour. Histopathological examination showed squamous cell carcinoma of vulva. In consequence, radical vulva removal with bilateral inguinal Sentinel node sampling was performed. A year later, recurrence in the vagina was diagnosed. After removal of the lesion the patient was referred for radiotherapy. In the examination preceding the radiation therapy, an enlarged, metastatic lymph node was found in the right groin.

Therapeutic strategy: (a) Irradiation of the pelvic lymph nodes with the dose up to $50.4 \mathrm{~Gy}$, the number of fractions -28 , fractional dose $-1.8 \mathrm{~Gy}$, (b) the area of the left groin with the dose up to $60 \mathrm{~Gy}$ and (c) the right groin and vulva with the dose up to $70 \mathrm{~Gy}$. Due to the right endoprosthesis, the first stage was performed by means of a tomotherapy machine. The remaining stages (b) and (c), which are not included in this paper, were performed using electron irradiation therapy by means of a conventional linear accelerator. Critical organs for the first stage of treatment: bladder, rectum, intestines, head of left femur and right endoprosthesis as the area of maximum dose reduction.

\section{Case 4 (tongue cancer)}

History: A 50-year-old woman reported a painful tongue swelling. A panendoscopic examination showed ulcerative lesions on the left lateral edge of the tongue. CT examination with contrast and MRI confirmed the presence of the tumour at the base of the tongue and two pathological lymph nodes. The biopsy showed squamous cell carcinoma. The tongue tumour cT2N2bMO was diagnosed. The patient underwent surgery and was referred for postoperative radiation therapy due to risk factors revealed in the pathology report such as extracapsular extension (ECE) in lymph nodes and close surgical margins.

Therapeutic strategy: The delivery of a dose of 66 Gy in 33 fractions to the high-risk area, $59.4 \mathrm{~Gy}$ in 33 fractions to the moderate risk area and a dose of $52.8 \mathrm{~Gy}$ in 33 fractions to the elective area. Protection of the spinal cord, brain stem, right parotid gland, larynx and mandible was prescribed.

\section{Case 5 (metastases to bones)}

History: A 35-year-old woman diagnosed with bone metastases from the primary breast tumour - T1cN2, treated with radio- and chemotherapy in 2004. After treating the primary lesion, complementary hormone therapy followed. In 2008 metastases to the sternum bone and the spine were found, and chemotherapy and the second hormone therapy were started. In 2010 metastases to L2, S1, S2 and the ischium bone were diagnosed.

Therapeutic strategy: The delivery of a total dose of 30 Gy in 10 fractions of irradiation. Protection of kidneys and the spinal cord was prescribed.

\section{Case 6 (advanced skin cancer)}

History: A 68-year-old man reported in 2006 with a $2 \mathrm{~cm}$ ulceration of an eyelid. The patient refused operation and dropped out of follow-up in 2009. An advanced skin cancer (T4NOMO) covering orbits, infiltrating paranasal sinuses and an eyeball was diagnosed. The functions of the eye were retained, with the visual field limited by the neoplasm. The patient accepted radiation therapy, and refused other options of treatment.

Therapeutic strategy: The delivery of a total dose of $60 \mathrm{~Gy}$ in 30 fractions to the tumour area. Critical organs: eyes, lenses, optic nerves, optic chiasm and brain stem. 
Table 1. Basic parameters used in the course of dose distribution planning for helical tomotherapy and conventional medical linear accelerator, for the following: 1 - anaplastic medulloblastoma, 2 - multifocal metastases to brain, 3 - vulva cancer, 4 - tongue cancer, 5 - metastases to bones, 6 - advanced skin cancer

\begin{tabular}{|c|c|c|c|c|c|c|c|c|}
\hline \multicolumn{2}{|c|}{ No of case } & \multicolumn{4}{|c|}{ Helical tomotherapy $(\mathrm{HT})$} & \multicolumn{3}{|c|}{ Conventional linear accelerator (CLA)* } \\
\hline \multirow{4}{*}{1} & & 1 phase & 2 phase & 3 phase & & 1 phase & 2 phase & 3 phase \\
\hline & FW: & 5 & 2.5 & 2.5 & Method: & 3 iso, 3DCRTC & 2 iso, $3 D C R T c$ & 1 iso, 3DCRTC \\
\hline & Pitch: & 0.287 & 0.287 & 0.287 & TNF: & 17 & 8 & 2 \\
\hline & MF: & 2.1 & 2.2 & 2.3 & Energy: & $6 \mathrm{MV}$ & $6 \mathrm{MV}$ & $6 \mathrm{MV}$ \\
\hline \multicolumn{9}{|c|}{ Simultaneus integrated boost } \\
\hline \multirow{3}{*}{2} & \multicolumn{2}{|l|}{ FW: } & \multicolumn{2}{|l|}{2.5} & Method: & \multicolumn{3}{|c|}{1 isocentre, IMRT, noncoplanar } \\
\hline & \multicolumn{2}{|l|}{ Pitch: } & 0.215 & & TNF: & \multicolumn{3}{|c|}{13} \\
\hline & MF: & & 5.0 & & Energy: & \multicolumn{3}{|c|}{$6 \mathrm{MV}$} \\
\hline \multirow{3}{*}{3} & FW: & & 2.5 & & Method: & \multicolumn{3}{|c|}{1 isocentre, IMRT, coplanar } \\
\hline & Pitch: & & 0.287 & & TNF: & \multicolumn{3}{|c|}{9} \\
\hline & \multicolumn{2}{|l|}{ MF: } & 2.4 & & Energy: & \multicolumn{3}{|c|}{$6 \mathrm{MV}$ and $20 \mathrm{MV}$} \\
\hline \multicolumn{9}{|c|}{ Simultaneus integrated boost } \\
\hline \multirow{3}{*}{4} & \multicolumn{2}{|l|}{ FW: } & 2.5 & & Method: & \multicolumn{3}{|c|}{1 isocentre, IMRT, noncoplanar } \\
\hline & \multicolumn{2}{|l|}{ Pitch: } & 0.287 & & TNF: & \multicolumn{3}{|c|}{7} \\
\hline & \multicolumn{2}{|l|}{ MF: } & 3.0 & & Energy: & \multicolumn{3}{|c|}{$6 \mathrm{MV}$} \\
\hline & \multicolumn{2}{|l|}{ FW: } & 2.5 & & Method: & & centres, IMRT, cO & \\
\hline 5 & Pitch: & & 0.215 & & TNF: & & 16 & \\
\hline & MF: & & 2.7 & & Energy: & & $20 \mathrm{MV}$ & \\
\hline & FW: & & 1 & & Method: & & ntre, IMRT, nonc & \\
\hline 6 & Pitch: & & 0.215 & & TNF: & & 6 & \\
\hline & MF: & & 1.8 & & Energy: & & $6 \mathrm{MV}$ & \\
\hline
\end{tabular}

Dose normalization: median PTV volume for each cases receives at least 100\% of the prescribed dose

$F W$ - field width in $\mathrm{cm}$

$M F$ - modulation factor

TNF - total number of fields

IMRT - intensity modulated radiotherapy

3DCRT - three dimensional conformal radiotherapy realized by coplanar fields

* for each case IMRT realized by sliding window technique using 120 multileaf collimator

Table 1 presents basic parameters used in the planning of dose distributions for helical tomotherapy and conventional medical accelerator.

Treatment plans were analysed with respect to the obtained dose distributions and the time necessary for their delivery. In the case of dose distributions, quality evaluation was performed using tomography scans, and quantity evaluation, during which both dose medians and maximum doses (measured at $1 \%$ of the volume of the analysed structure) for critical organs were compared.

For the planning target volumes (PTV), the homogeneity of dose distribution was analysed. In order to do that, moderate doses, standard deviations and the analysis of differential histograms were used. The analysis of the therapy time involved reviewing the total time in which the treatment dose was delivered, taking into account the breaks between the delivery of the dose by the consecutive treatment fields. It was assumed that the time necessary to change the posi- tion of MLC leaves for fields divided during optimization was $5 \mathrm{~s}$. The change of treatment fields without a change in table positioning is $15 \mathrm{~s}$ and with such a change is $60 \mathrm{~s}$. In the case of a fraction during which more than one treatment plan is carried out, it was assumed that the time necessary for the change of the isocentre is $180 \mathrm{~s}$.

The application of exporting the tomotherapy plans to the outside treatment planning system enabled to compare the dose distributions in one treatment planning system Eclipse (Varian Inc).

\section{Results}

Figure 1 shows dose distributions for (a) conventional linear accelerator (CLA) and (b) helical tomotherapy HT. In order to present dose distributions, CT scans showing target volume and critical organs were chosen. Clinical cases presented in Figure 1 were marked as follows: 1 - anaplastic medulloblastoma, 2 - multifocal metastases to brain, 3-vul- 

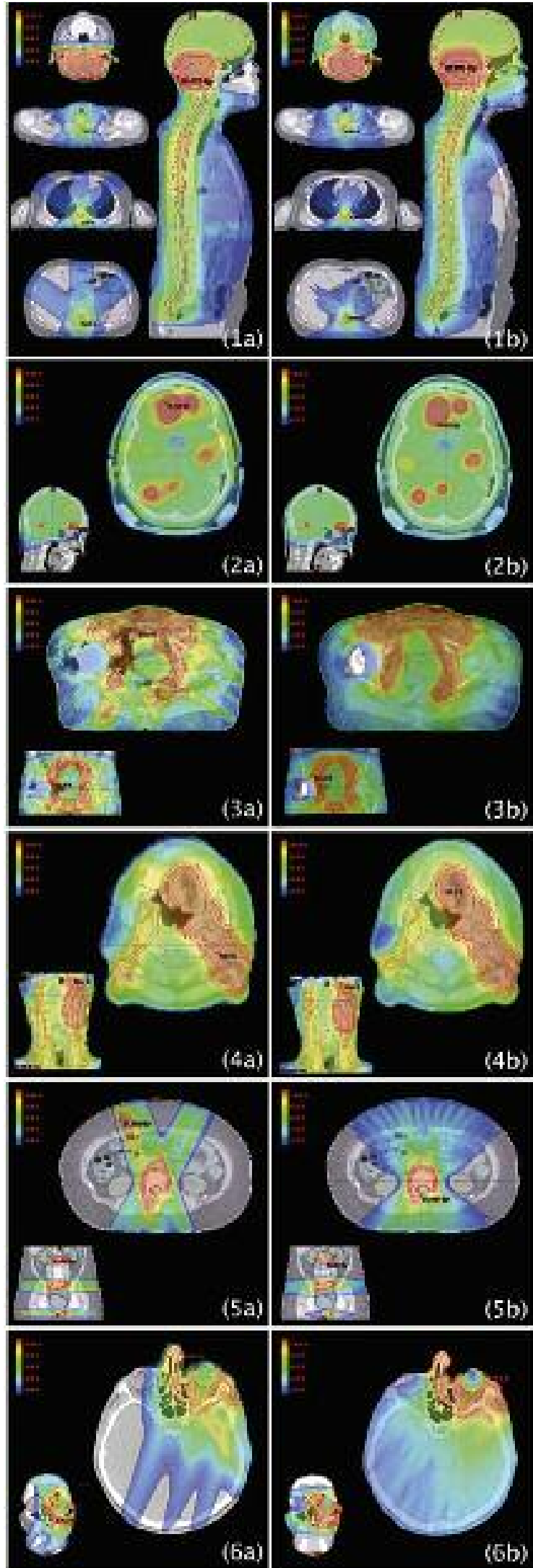

Fig. 1. Comparison of dose distributions on tomography scans for plans implemented using (a) conventional linear accelerator and (b) helical tomotherapy. Individual clinical cases have been marked with the following numbers: 1 - anaplastic medulloblastoma, 2 - multifocal metastases to brain, 3 - vulva cancer, 4 - tongue cancer, 5 - metastases to bones, 6 - advanced skin cancer va cancer, 4-tongue cancer, 5 - metastases to bones, 6-advanced skin cancer. These denotations will be consistently used in the following part of this paper.

Table 2 presents the time necessary to deliver a fractional dose for individual cases, in the case of CLA and HT respectively. Additionally, the values shown in Table 2 do not take into account the time needed to perform image verification.

Following the analysis of dose distributions in the planning target volume (PTV), decrease of the dose homogeneity in the case of the CLA plan in relation to HT was observed. Dose homogeneity in PTV was determined on the basis of standard deviation (SD). In most cases (1, 2, 3, 5), SD was twice as high in the case of CLA plans than HT plans. For case 6, SD obtained for CLA was four times higher than SD for HT. Only case 4 displayed no significant differences between standard deviations. The difference of standard deviations in the case of CLA and HT was in the range of $10 \%$ calculated in relation to a smaller SD obtained for HT. However, case 4 exhibited a bigger dose median in an elective irradiation area in the case of the CLA plan in comparison to the HT plan. The dose median for HT was 54.1 Gy. In the case of CLA, it was higher by 2 Gy (56.1 Gy). Moreover, a prescribed dose, determined by a radiation therapist, in this target volume was $52.8 \mathrm{~Gy}$. A similar phenomenon was observed in case 1 in the target volume, covering the spinal cord and the whole brain. The HT dose median was 35.8 Gy and CLA - 36.9 Gy. The prescribed dose in this target volume was $35.1 \mathrm{~Gy}$. Figure 2 shows differential histograms of dose distribution in PTV area for all clinical cases. The distribution of blue areas denoting HT and yellow ones denoting CLA (Fig. 2) depicts a fall in dose homogeneity for $\mathrm{CLA}$ in relation to $\mathrm{HT}$. Moreover, in Fig. 2, the prescribed dose (black line), determined before the start of the planning, was marked on each graph. A rise of dose median for CLA in relation to HT in cases 1 and 4 is presented in Graphs 1 and 2 respectively as a shift of the CLA histogram (red line) in relation to the HT histogram (blue line) and the prescribed dose (black line). On graphs 1, 2 and 4 (Fig. 2, cases 1, 2 and 4 respectively), the $x$-axis (presenting percent values of doses) was shortened. This enabled a better visualization of the dif-

Table 2. Time in seconds necessary for delivering fractional dose for helical tomotherapy and conventional medical linear accelerator, for the following: 1 - anaplastic medulloblastoma, 2 - multifocal metastases to brain, 3 - vulva cancer, 4 - tongue cancer, 5 - metastases to bones, 6 - advanced skin cancer

\begin{tabular}{|lcc|}
\hline No of case & \multicolumn{2}{c|}{ Time of the fraction dose delivery [s] } \\
\hline & $H T$ & CLA \\
\hline 1 & 646 & 878 \\
& 602 & 350 \\
\hline 2 & 202 & 48 \\
\hline 3 & 542 & 1191 \\
\hline 4 & 493 & 649 \\
\hline 5 & 441 & 660 \\
\hline 6 & 1480 & 1241 \\
\hline
\end{tabular}



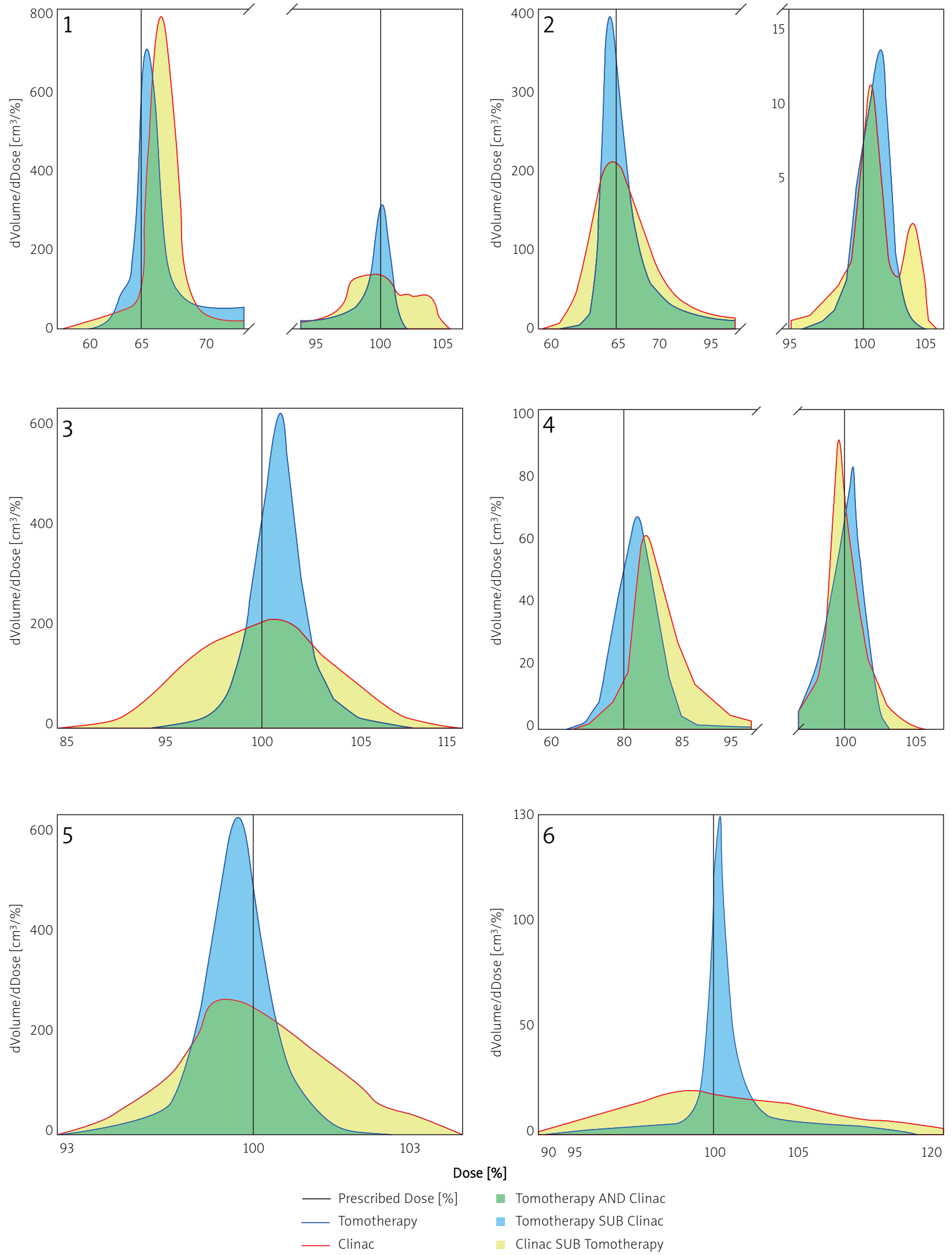

Fig. 2. Differential histogram of dose distribution in planning target volume (PTV) of helical tomotherapy (blue line) and radiotherapy delivered by a conventional linear accelerator (red line) Clinical cases were marked as follows: 1 - anaplastic medulloblastoma, 2 - multifocal metastases to brain, 3 - vulva cancer, 4 - tongue cancer, 5 - metastases to bones, 6 - advanced skin cancer. Black line on each graph denotes expected value of therapeutic dose. The green area denotes overlapping part of the histograms for tomotherapy and a conventional linear accelerator, yellow area belongs only to the histogram for a conventional linear accelerator, and blue area belongs only to the histogram for tomotherapy 
Table 3. Comparison of the median dose and maximum dose in organ at risk for plans implemented using: (a) conventional linear accelerator and (b) helical tomotherapy. Clinical cases have been marked with the following numbers: 1 - anaplastic medulloblastoma, 2 - multifocal metastases to brain, 3 - vulva cancer, 4 - tongue cancer, 5 - metastases to bones, 6 - advanced skin cancer

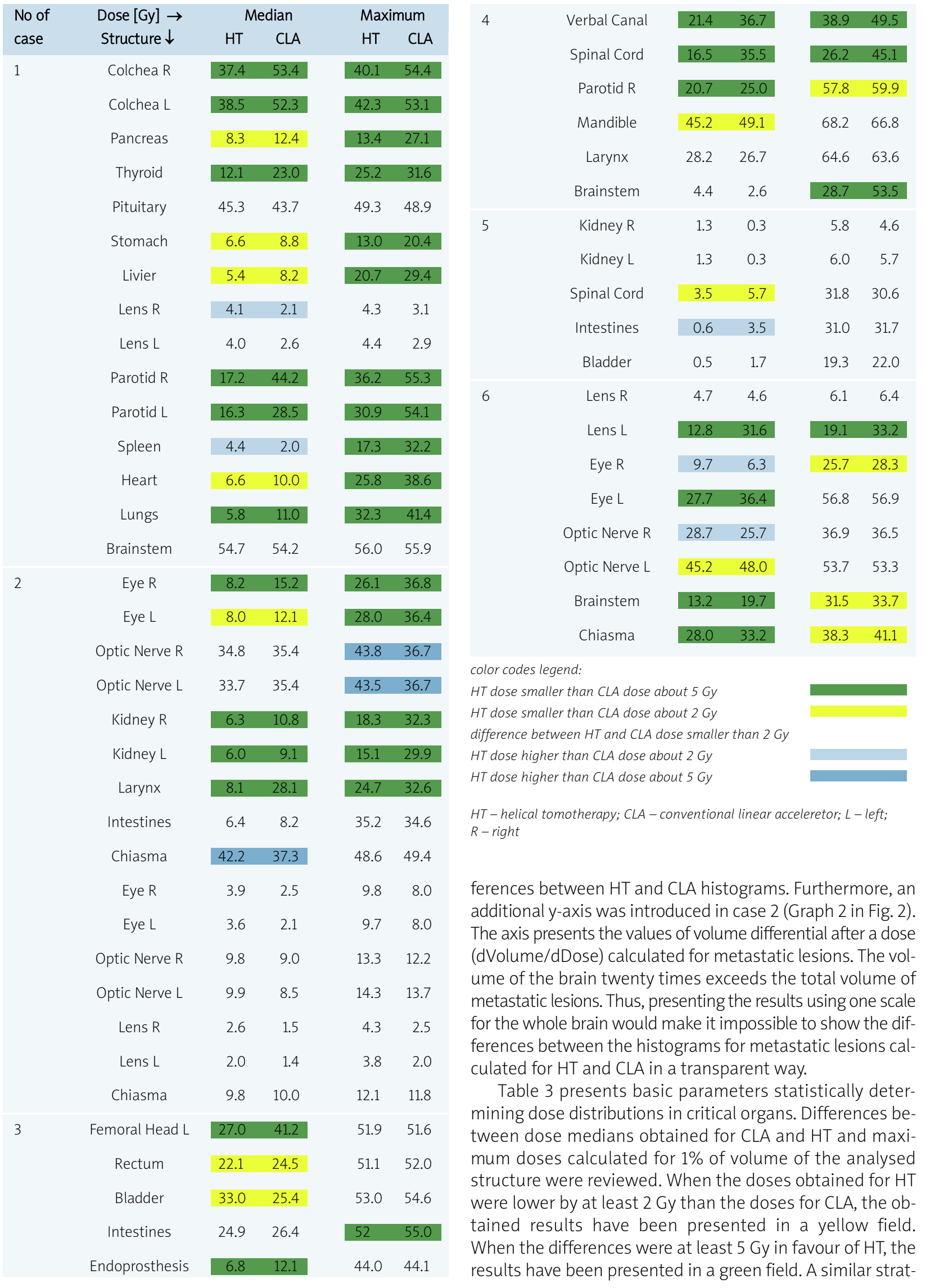



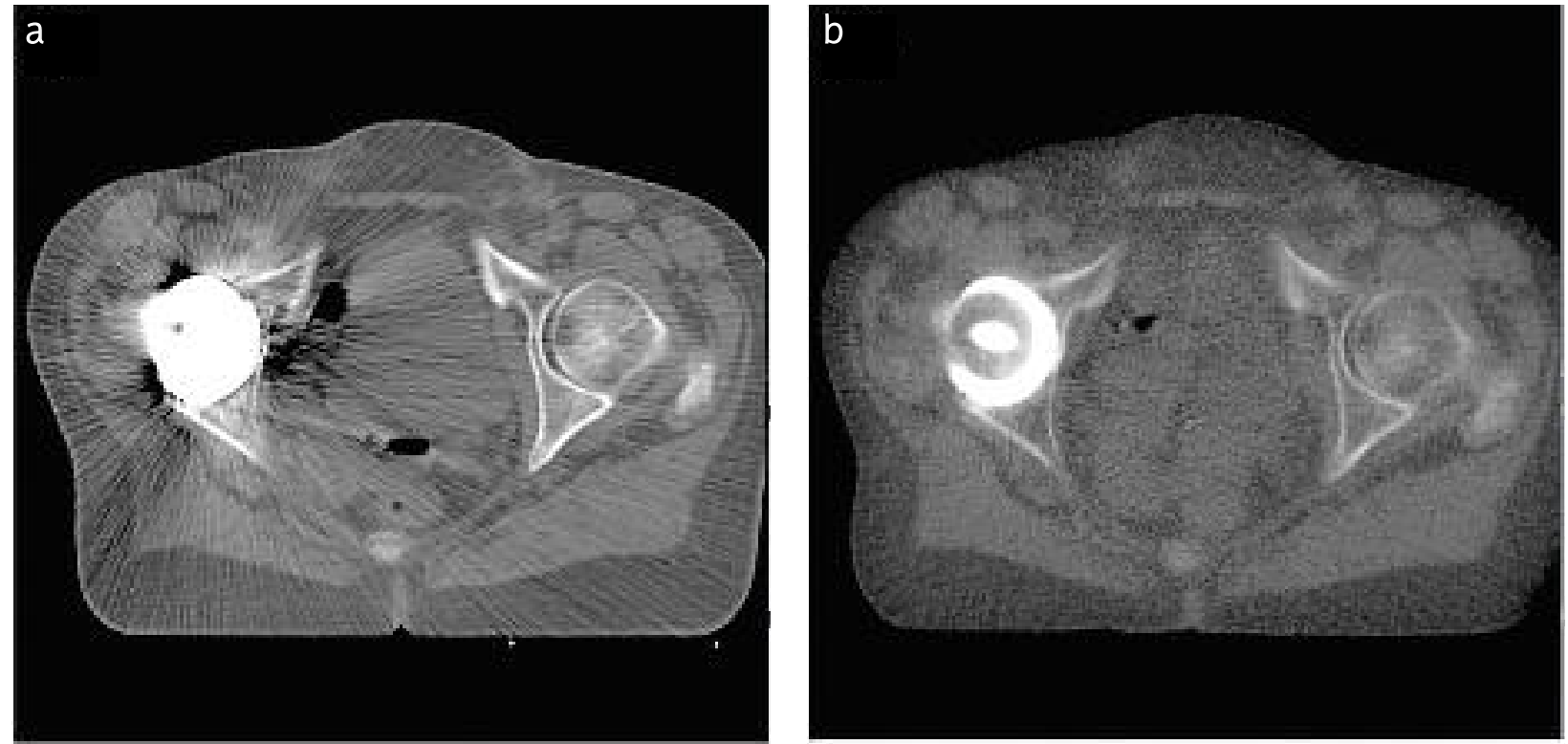

Fig. 3. Tomography scans of the pelvis area with endoprosthesis taken by: a) computed tomography; b) helical tomography. In both cases the same imaging window was used

egy was employed for the situation when doses for CLA were lower than those for HT. Light blue fields and dark blue fields denote results which differ by at least 2 Gy and 5 Gy, respectively.

\section{Discussion}

Helical tomotherapy $(\mathrm{HT})$ is one of the methods of radiotherapeutic treatment. This method enables treatment implementation for a wide spectrum of clinical cases, beginning with very small neoplastic lesions located in the brain $[29,30]$ or in the whole body $[31,32]$, treated with conventional stereotactic methods or radiation surgery, and ending with vast target volumes such as anaplastic medulloblastoma, total body irradiation (TBI) or total marrow irradiation (TMI) $[33,34]$. In these case using a conventional linear accelerator (CLA) requires special therapeutic procedures, often involving the modification of radiation geometry (using nonstandard treatment distances, specially designed treatment tables, etc.) $[35,36]$. The wide variety of clinical uses of helical tomotherapy results directly from the method of dose delivery. It differs significantly from the classic method, typical of conventional linear accelerators. In Poland, at the turn of 2011 a tomotherapy machine was installed. Therefore, one of the aims of this publication was to present the method in which such a machine operates. Taking into consideration the difference in dose delivery and the consequent different method of treatment plan preparation, we also discuss basic issues concerning the treatment planning process.

In order to demonstrate the possibilities of helical tomotherapy, treatment plans for six different clinical cases have been presented. They have been compared with corresponding plans prepared for a conventional linear accelerator. The authors decided to avoid limiting the presentation to cases representing only one clinical location since there are many such specialist reports which compare helical tomotherapy with radiotherapy delivered using CLA [37-44]. The majority of quoted papers emphasize the increase in conformity of dose distributions for tomotherapy treatment in relation to compared methods. The increase in conformity is understood here as a rise in dose homogeneity in the area of irradiation and reduction of doses in critical organs. Nevertheless, none of these papers presents the wide variety of clinical uses of the tomotherapy machine resulting from its unique technological solutions.

The first case reported in this publication was the irradiation of anaplastic medulloblastoma (Fig. 1). Helical tomotherapy as the only method offers the possibility to irradiate the whole brain together with the spinal cord during one working period of the machine. The source rotates around an irradiated area following a spiral path. Thus, a treatment field can be formed of total size of $160 \mathrm{~cm}$ measured along the patient's longitudinal axis. In the case of conventional linear accelerators, the implementation of such treatment requires the use of a number of treatment fields divided into at least three target volumes (brain and cervical part of the spine, thoracic part of the spine and lumbar-sacral part of the spine). Application of the total therapeutic dose requires at least two changes in treatment fields' set-up. This is the consequence of the length of the field which can be obtained with a conventional linear accelerator $(40 \mathrm{~cm})$. The change in set-up may result in underdosed areas and overdosed ones in regions where the fields overlap [45].

When comparing the obtained results of dose distributions, it should be noted that in the case of a tomotherapy plan there was a significant reduction of doses in most critical organs (Table 3). Moreover, we obtained a greater dose homogeneity in target volume delineated for a residual lesion adjoining the brain stem (Fig. 2, Graph 1, high dose range 95-105\%) without an increase of dose median in the brain area and the spinal cord (Fig. 2, Graph 1, dose range 60-70\%). Furthermore, the correct choice of parameters characterizing the method of dose delivery by a tomotherapy machine 
(Table 1) allows one to obtain, in the first stage of treatment (cranio-spinal axis), irradiation times comparable to those obtained using a conventional linear accelerator.

Case 2 shows a different clinical situation, which requires the simultaneous use of small stereotactic fields (metastatic lesions) and standard irradiation fields (whole-brain irradiation). In the case of a conventional linear accelerator, it is possible to deliver radiation in two ways. In one, the therapy can be split into two stages of treatment: during the first stage the whole brain is irradiated while the second is devoted to stereotactic treatment (a stereotactic system is required). In the other, an attempt can be made to carry out a multi-field and non-coplanar IMRT plan which assumes the irradiation of the whole brain with a simultaneous integrated boost dose to metastatic lesions (SIB, an example discussed in the paper). In the case of tomotherapy, combining minimum jaw width $(1 \mathrm{~cm})$ and low pitch (0.215) makes SIB treatment possible. It is worth pointing out that for both $\mathrm{HT}$ and CLA, reduction of doses in critical organs is comparable (Table 3). However, the application of tomotherapy leads to the increase in homogeneity of dose distributions for the brain and metastatic lesions (Fig. 1 and Graph 2 shown in Fig. 2). Moreover, the time of tomotherapy treatment implementation is twice as short as the time for a conventional linear accelerator (Table 2).

The next case (case 3, vulval cancer) illustrates the possibility of using additional functionality of a tomotherapy machine. Cancer of the vulva is successfully treated by the IMRT method using a conventional linear accelerator. Nevertheless, in the case of patients who have an endoprosthesis of a hip joint, doses in both an implant area and in critical organs, such as the healthy head of a femur, the bladder, intestines and the rectum, need special reduction. Direct adhesion of the target volume to an implant makes the clinical situation even more complicated.

Furthermore, the quality of CT scans used in treatment planning is lower due to the artefacts which are created during the imaging of the area with an endoprosthesis. This leads to a greater error in the delineation of both target volume and critical organs. The use of these scans makes the calculations of dose distributions also burdened with error. In order to make a correction, the algorithms which carry out the computing take into account the correlation between grey scale expressed in $\mathrm{HU}$ and densities of illustrated tissues. On the other hand, HT offers the possibility of taking MVCT scans. The quality of MVCT is lower than that of CT; however, MVCT images are artefact-free. Figure 3 shows a CT scan with a visible endoprosthesis and artefacts (a) and a corresponding MVCT scan (b).

The use of both sets of scans - CT and MVCT - in the process of delineation and calculation of dose distributions during tomotherapy planning results in a significant reduction of inaccuracies with which plans for a conventional linear accelerator are burdened. What is more, referring to the comparison of dose distributions, application of tomotherapy leads to an increase of dose homogeneity in target volume (Figure 2, Graph 3) and a reduction of doses in critical organs (Table 3).

The next two clinical cases (cancer of the tongue and metastases to bones) confirm the increase in conformity of dose distribution for tomotherapy treatment in relation to therapy implemented using conventional linear accelerators. In the case of tongue cancer (Graph 4, Fig. 1) the main factor influencing the increase in conformity is a significant reduction of doses in critical organs (Table 3) while retaining homogeneity of dose distributions in target volumes, comparable to that obtained for conventional linear accelerators (Graph 4, Fig. 2). In the case of metastases to bones, the increase of conformity is the result of obtaining better homogeneity of dose distributions in target volume (Graph 5, Fig. 2). The reduction of doses in the spinal cord should also be noted (Graph 5 in Fig. 1).

The last clinical case is advanced skin neoplasm covering an orbit, an eyelid, and paranasal sinuses. The use of tomotherapy allowed a considerable reduction of doses in critical organs located in the direct vicinity of the target volume (left eye, lens, optic nerve and optic chiasm and brain stem) and significantly higher homogeneity of dose in the radiation target area in relation to CLA (Graph 6 in Fig. 2). However, there was an increase in doses absorbed by the remaining critical organs located further away from the radiation target area, i.e. the right eye and right optic nerve (Table 3).

To conclude, helical tomotherapy makes it possible to obtain highly conformal dose distributions for treatments carried out for both vast target volumes (anaplastic medulloblastoma) and small areas, treated conventionally with stereotactic methods (metastases to the brain). The application of imaging technology that a tomotherapy machine offers, in some cases (vulval cancer), increases the precision of delineating the radiation target area and critical organs, and reduces the error in dose calculation resulting from taking into account incorrect HU values (artefacts). The analysis of dose distributions for the remaining cases discussed in the paper confirms the increase of treatment conformity through dose reduction in critical organs (tongue cancer), the increase of homogeneity of dose distribution in a radiation target area (metastases to bones) or through both these effects (advanced skin cancer).

\section{References}

1. Kukołowicz PF. Charakterystyka wiązek terapeutycznych fotonów i elektronów. RTA, Kielce 2001.

2. Piotrowski T, Kazmierska J. Tomoterapia spiralna. W: Skuteczna ochrona radiologiczna w medycynie. Kubicka M, Barczyk J (ed.). Verlag Dashofer, Warszawa 2008.

3. Galvin JM, Smith AR, Moeller RD, et al. Evaluation of multileaf collimator design for a photon beam. Int J Radiat Oncol Biol Phys 1992; 23: 789-801.

4. Brahme A. Optimal setting of multileaf collimators in stationary beam radiation therapy. Strahlenther Onkol 1988; 164: 343-50.

5. Milecki P, Stryczyńska G, Nawrocki S, Iżycki D. Radioterapia konformalna 3-D - stan obecny i perspektywy. Wspolczesna Onkol 2000; 1: 30-2.

6. Kallman P, Lind B, Eklof A, Brahme A. Shaping of arbitrary dose distributions by dynamic multileaf collimation. Phys Med Biol 1988; 33: 1291-300.

7. Grządziel A, Ślosarek K, Rutkowski R, Rembielak A. IMRT - new standards in treatment planning. Rep Pract Oncol Radiother 2001; 6: 29-30.

8. Maciejewski B, Malicki J, Ślosarek K, Drzewiecka B, Radiobiological rationale for advantages and limitations of IMRT in clinical practice. Nowotwory 2001; 51: 355-64. 
9. Bindhu J, Supe S, Pawar Y. Intensity modulated radiotherapy (imrt) the white, black and grey: a clinical perspective. Rep Pract Oncol Radiother 2009; 14: 95-103.

10. Ziółkowska E, Reszke J, Morgaś T, Drzewiecka B, Makarewicz R. Rola IMRT $w$ redukcji dawki promieniowania $w$ odbytnicy $w$ trakcie radykalnej radioterapii raka gruczołu krokowego. Wspolczesna Onkol 2005; 9: 296-9.

11. Idasiak A, Wolny E, Wydmański J, Hawrylewicz L, Miszczyk L. Zastosowanie konformalnej radioterapii i modulacji intensywności wiąz ki w planowaniu pooperacyjnej radioterapii chorych na raka trzustki. Wspolczesna Onkol 2004; 8: 347-52.

12. Sas-Korczyńska B, Śładowska A, Rozwadowska-Bogusz B, Dyczek S, Lesiak J, Kokoszka A, Korzeniowski S. Comparison between inten sity modulated radiotherapy (IMRT) and 3D tangential beams tech nique used in patients with early-stage breast cancer who received breast-conserving therapy. Rep Pract Oncol Radiother 2010; 15 79-86.

13. Otto K. Volumetric modulated arc therapy: IMRT in a single gantry arc. Med Phys 2008; 35: 310-7.

14. Bedford JL. Treatment planning for volumetric modulated arc ther apy. Med Phys 2009; 36: 5128-38.

15. Pesce GA, Clivio A, Cozzi L, Nicolini G, Richetti A, Salati E, Valli M, Vanetti E, Fogliata A. Early clinical experience of radiotherapy of prostate can cer with volumetric modulated arc therapy. Radiat Oncol 2010; 5: 54

16. Doornaert P, Verbakel WF, Bieker M, Slotman BJ, Senan S. RapidArc planning and delivery in patients with locally advanced head-and neck cancer undergoing chemoradiotherapy. Int J Radiat Oncol Bio Phys 2011; 79: 429-35.

17. Popple RA, Fiveash JB, Brezovich IA, Bonner JA. RapidArc radiation ther apy: first year experience at the University of Alabama at Birming ham. Int J Radiat Oncol Biol Phys 2010; 77: 932-41.

18. Welsh JS, Patel RR, Ritter MA, Harari P, Mackie TR, Mehta MP. Helical tomotherapy: an innovative technology and approach to radiation therapy. Technol Canc Res Treat 2002; 1: 55-63.

19. Mackie TR. History of tomotherapy. Phys Med Biol 2006; 51: 427-453.

20. Dejean C, Kantor G, Henriques de Figueiredo B, et al. Helical tomotherapy: description and clinical applications. Bull Cancer 2010; 97: 783-9.

21. Reynders T, Tournel K, De Coninck P, et al. Dosimetric assessment of static and helical TomoTherapy in the clinical implementation of breast cancer treatments. Radiother Oncol 2009; 93: 71-9.

22. Sterzing F, Uhl M, Hauswald H, et al. Dynamic jaws and dynamic couch in helical tomotherapy. Int J Radiat Oncol Biol Phys 2010; 76: 1266-73.

23. Sen A, West MK. Commissioning experience and quality assurance of helical tomotherapy machines. J Med Phys 2009; 34: 194-9.

24. Shah AP, Langen KM, Ruchala KJ, Cox A, Kupelian PA, Meeks SL. Patient dose from megavoltage computed tomography imaging. Int J Radiat Oncol Biol Phys 2008; 70: 1579-87.

25. Welsh JS, Lock M, Harari PM, et al. Clinical implementation of adaptive helical tomotherapy: a unique approach to image-guided intensity modulated radiotherapy. Technol Cancer Res Treat 2006; 5: 465-79.

26. Brahme A. Optimization of stationary and moving beam radiation therapy techniques. Radiother Oncol 1988; 12: 129-40.

27. Holmes TW, Mackie TR, Reckwerdt P. An iterative filtered backprojection inverse treatment planning algorithm for tomotherapy. Int J Radiat Oncol Biol Phys 1995; 32: 1215-25.

28. Kissick MW, Fenwick J, James JA, et al. The helical tomotherapy thread effect. Med Phys 2005; 32: 1414-23.

29. Tomita N, Kodaira T, Tachibana H, Nakamura T, Nakahara R, Inokuchi H, Shibamoto Y. Helical tomotherapy for brain metastases: dosimetric evaluation of treatment plans and early clinical results. Technol Cancer Res Treat 2008; 7: 417-24.

30. Sterzing F, Welzel T, Sroka-Perez G, Schubert K, Debus J, Herfarth KK. Reirradiation of multiple brain metastases with helical tomotherapy. A multifocal simultaneous integrated boost for eight or more lesions. Strahlenther Onkol 2009; 185: 89-93.

31. Chawla S, Abu-Aita R, Philip A, Lundquist T, Okunieff P, Milano MT. Stereotactic radiosurgery for spinal metastases: case report and re view of treatment options. Bone 2009; 45: 817-21.

32. Kim JY, Kay CS, Kim YS, Jang JW, Bae SH, Choi JY, Yoon SK, Kim KJ. He lical tomotherapy for simultaneous multitarget radiotherapy for pulmonary metastasis. Int J Radiat Oncol Biol Phys 2009; 75: 703-10.
33. Peñagarícano J, Moros E, Corry P, Saylors R, Ratanatharathorn V. Pediatric craniospinal axis irradiation with helical tomotherapy: patient outcome and lack of acute pulmonary toxicity. Int J Radiat Oncol Biol Phys 2009; 75: 1155-61.

34. Zhuang AH, Liu A, Schultheiss TE, Wong JY. Dosimetric study and verification of total body irradiation using helical tomotherapy and its comparison to extended SSD technique. Med Dosim 2010; 35: 243-9.

35. Malicki J. Accuracy of dose determination during total body irradiation. Strahlenter Onkol 1999; 175: 208-12.

36. Malicki J, Wachowiak J, Kosicka G, Stryczyńska G, Nowak A, Pracz J. Total body irradiation before bone marrow transplantation: aims and results. Adv Exp Med Biol 2001; 495: 277-82

37. Chen AM, Lee NY, Yang CC, Liu T, Narayan S, Vijayakumar S, Purdy JA. Comparison of intensity-modulated radiotherapy using helical tomotherapy and segmental multileaf collimator-based techniques for nasopharyngeal carcinoma: dosimetric analysis incorporating quality assurance guidelines from RTOG 0225. Technol Cancer Res Treat 2010; 9: 291-8

38. Zhang X, Penagaricano J, Moros EG, Corry PM, Yan Y, Ratanatharathorn $V$. Dosimetric comparison of helical tomotherapy and linac-IMRT treatment plans for head and neck cancer patients. Med Dosim 2010; 35: 264-8.

39. Kim S, Lee IJ, Kim YB, Koom WS, Jeon BC, Lee CG, Kim GE, Keum KC. A comparison of treatment plans using linac-based intensitymodulated radiation therapy and helical tomotherapy for maxillary sinus carcinoma. Technol Cancer Res Treat 2009; 8: 257-63.

40. Tomita N, Kodaira T, Tachibana H, Nakamura T, Nakahara R, Inokuchi H, Mizoguchi N, Takada A. A comparison of radiation treatment plans using IMRT with helical tomotherapy and 3D conformal radiotherapy for nasal natural killer/T-cell lymphoma. Br J Radiol 2009; 82: 756-63.

41. Kim YB, Kim JH, Jeong KK, Seong J, Suh CO, Kim GE. Dosimetric comparisons of three-dimensional conformal radiotherapy, intensitymodulated radiotherapy, and helical tomotherapy in whole abdominopelvic radiotherapy for gynecologic malignancy. Technol Cancer Res Treat 2009; 8: 369-77.

42. Kunos CA, Dobbins DC, Kulasekere R, Latimer B, Kinsella TJ. Comparison of helical tomotherapy versus conventional radiation to deliver craniospinal radiation. Technol Cancer Res Treat 2008; 7: 227-33.

43. Cattaneo GM, Dell'oca I, Broggi S, et al. Treatment planning comparison between conformal radiotherapy and helical tomotherapy in the case of locally advanced-stage NSCLC. Radiother Oncol 2008; 88: 310-8.

44. Alongi F, Fiorino C, Cozzarini C, Broggi S, Perna L, Cattaneo GM, Calandrino R, Di Muzio N. IMRT significantly reduces acute toxicity of whole-pelvis irradiation in patients treated with post-operative adjuvant or salvage radiotherapy after radical prostatectomy. Radiother Oncol 2009; 93: 207-12.

45. Slampa P, Zitterbart K, Dusek L, et al. Craniospinal irradiation of medulloblastoma in the supine position. Rep Pract Oncol Radiother 2006; 11: 25-272.

\section{Address for correspondence}

Tomasz Piotrowski PhD

Zakład Fizyki Medycznej

Wielkopolskie Centrum Onkologii

ul. Garbary 15

61-866 Poznań

e-mail: tomasz.piotrowski@me.com

tel. +48618850763

\section{Submitted: $\quad 13.03 .2011$}

Accepted: $\quad$ 18.01.2012 